\title{
Ophthalmology Residents' Experience with Complex Cataract Surgery at a VA Hospital over 10 Years
}

\author{
Colleen Maturana, MD ${ }^{1,2}$ Paul Lee, MD ${ }^{1,3}$ Douglas Fredrick, MD ${ }^{1}$ Nisha Chadha, MD ${ }^{1}$
}

${ }^{1}$ Department of Ophthalmology, Icahn School of Medicine at Mount Sinai/New York Eye and Ear, Eye and Vision Research Institute, New York, New York

2 Department of Ophthalmology, Weill Cornell Medicine, New York, New York

${ }^{3}$ Department of Ophthalmology, James J. Peters VA Medical Center, Bronx, New York

J Acad Ophthalmol 2020;12:e36-e40.

\begin{abstract}
Address for correspondence Nisha Chadha, MD, Department of Ophthalmology, Icahn School of Medicine at Mount Sinai/New York Eye and Ear, Eye and Vision Research Institute, 17 E 102nd Street, 8th Floor West, New York, NY 10029 (e-mail: Nisha.chadha@mssm.edu).
\end{abstract}

Cataract is the leading cause of visual impairment in the United States. More individuals are affected by cataracts than by age-related macular degeneration, glaucoma, and diabetic retinopathy combined. ${ }^{1}$ In 2010 , there were an estimated 24.4 million Americans with cataracts and this number is estimated to almost double to 50 million by $2050 .^{2}$ Cataract surgery is the most common procedure performed by ophthalmologists. In the United State alone in 2010, there were 6,353 cataract surgeries per 1 million people performed. ${ }^{3}$ In order make certain that this burgeoning population of individuals requiring cataract surgery receive the safest surgery with the optimal visual outcomes, academic training received

September 23, 2019

accepted

March 1, 2020
DOI https://doi.org/

10.1055/s-0040-1709674. ISSN 2475-4757.
Copyright $\odot 2020$ by Thieme Medical

Publishers, Inc., 333 Seventh Avenue, New York, NY 10001, USA. Tel: +1(212) 760-0888
License terms

()ㅇ (1) $\Theta \circledast$ 
programs must graduate residents who possess excellent surgical skills and clinical judgment.

The ideal method for training residents to become independent, confident, and capable surgeons is continuously being evaluated and refined. The Accreditation Council for Graduate Medical Education (ACGME), a nonprofit organization that evaluates and accredits medical residency training programs in the United States, has outlined a required minimum number of procedures to graduate. ${ }^{4}$ These numbers are meant to ensure that graduates are competent to enter into independent practice without direct supervision. The current minimum required number of phacoemulsification cataract surgeries is 86 . The National Resident Report from 2017 reported an average of 186.4 and median of 183 phacoemulsification cases performed as primary surgeon among residents completing programs in 2016 to $2017 .^{5}$ However, specification of routine versus complex cataract surgery is not required or recorded in the ACGME case logs.

It is generally accepted that there is a positive correlation between quantity of a procedure performed by a surgeon and that surgeon's outcomes. However, there is variability depending on individual procedures and conditions. ${ }^{6}$ A review of patient outcomes based on cataract surgeon volumes found a low adverse event rate after 50 procedures per year, but this rate declined even more with increasing volume. ${ }^{7}$

To be able to demonstrate and attain competence in cataract surgery, residents must not only perform a sufficient number of surgeries but these surgeries should also be diverse in difficulty and technique. The purpose of this study was to evaluate third-year resident's experience with complex cataract surgery at a VA Medical Center where a significant portion of the cataract surgery experience, approximately $50 \%$, is gained in their final year of training.

\section{Methods}

A chart review of resident cataract surgeries was performed from July 1, 2007,to June 30, 2017, at the James J. Peters VA Medical Center in The Bronx, NY. Surgeries were categorized by Current Procedural Terminology code with 66984 indicating routine cataract surgery and 66982 indicating complex cataract surgery. Correct categorization was confirmed by review of operative report, so as to not miss complex cases that were unintentionally coded as routine, and reason for complex categorization was recorded, as well as the use and type of nonstandard device or technique. Cases complicated by posterior capsule rupture and/or requiring anterior vitrectomy were excluded, as the purpose was to review complex, rather than complicated, cataract procedures. This study was determined to be exempt by the Icahn School of Medicine at Mount Sinai Institutional Review Board.

\section{Results}

A total of 2,543 cataract surgeries were performed by 40 different residents from one residency program over a 10year period at the James J. Peters VA Hospital. With four residents per year, each resident spent 3 months of their
Table 1 Number of cataract surgeries from 2007 to 2017

\begin{tabular}{|l|l|}
\hline Total cataract cases & 2,543 \\
\hline Average total cataract cases per resident & 63 \\
\hline Range of total cataract cases per resident & $28-101$ \\
\hline Total complex cataract cases & 114 \\
\hline Average complex cataract cases per resident & 2 \\
\hline Range complex cataract cases per resident & $0-10$ \\
\hline
\end{tabular}

third year at this hospital where they performed approximately $50 \%$ of their total cataract volume. All cataract surgery is performed by residents under the direct supervision of an attending. Of note, results for 2007 include only data from July to December and results for 2017 include only data from January to June because academic year, rather than the calendar year, was used for this study.

- Table 1 is representative of approximately 50\% of the surgery that residents performed during their third year of training. Out of all cataract surgery performed, $95.5 \%$ of the cases were routine, with the other $4.5 \%$ of cases being complex. Average cases per resident were 63 routine (range: 28-101) and 2 complex (range: 0-10). Reasons for complex categorization included intraoperative floppy iris syndrome (35.8\%), miosis (38.4\%), zonular instability (9.6\%), mature cataract (7\%), posterior synechiae (7.8\%), and posterior capsular plaque (1.8\%) as shown in - Table 2. Mature cataract was defined as either a white cataract or grade $4+$ nuclear sclerosis for which trypan blue was typically utilized. Miosis and intraoperative floppy iris syndrome were found to be the most common reasons for complex categorization. There was no evident diagnosis to explain poor dilation in most of the miosis patients, but the patients with intraoperative floppy iris syndrome were on an $\alpha-1$ antagonist medication.

Types of nonstandard techniques/devices included iris hooks (65.3\%), Malyugin ring (8.5\%), extracapsular cataract extraction (6.8\%), synechiolysis (7.6\%), capsular tension ring (9.3\%), mechanical iris dilation ( $0.8 \%)$, and primary posterior continuous curvilinear capsulorhexis (1.7\%; - Fig. 1). Techniques used for pupil expansion were the most common. The two most infrequent techniques were primary posterior capsulorhexis, used twice for posterior capsule fibrosis, and mechanical dilation of the pupil, used once for miosis. Very few cases required capsular tension rings for zonular instability. The

Table 2 Reasons for complex categorization

\begin{tabular}{|l|l|l|}
\hline Reason & Frequency & \% of total \\
\hline IFIS & 42 & 35.8 \\
\hline Miosis & 45 & 38.4 \\
\hline Zonular instability & 11 & 9.6 \\
\hline Posterior synechiae & 9 & 7.8 \\
\hline Mature cataract & 8 & 7.0 \\
\hline Posterior capsule plaque & 2 & 1.8 \\
\hline
\end{tabular}

Abbreviation: IFIS, intraoperative floppy iris syndrome. 


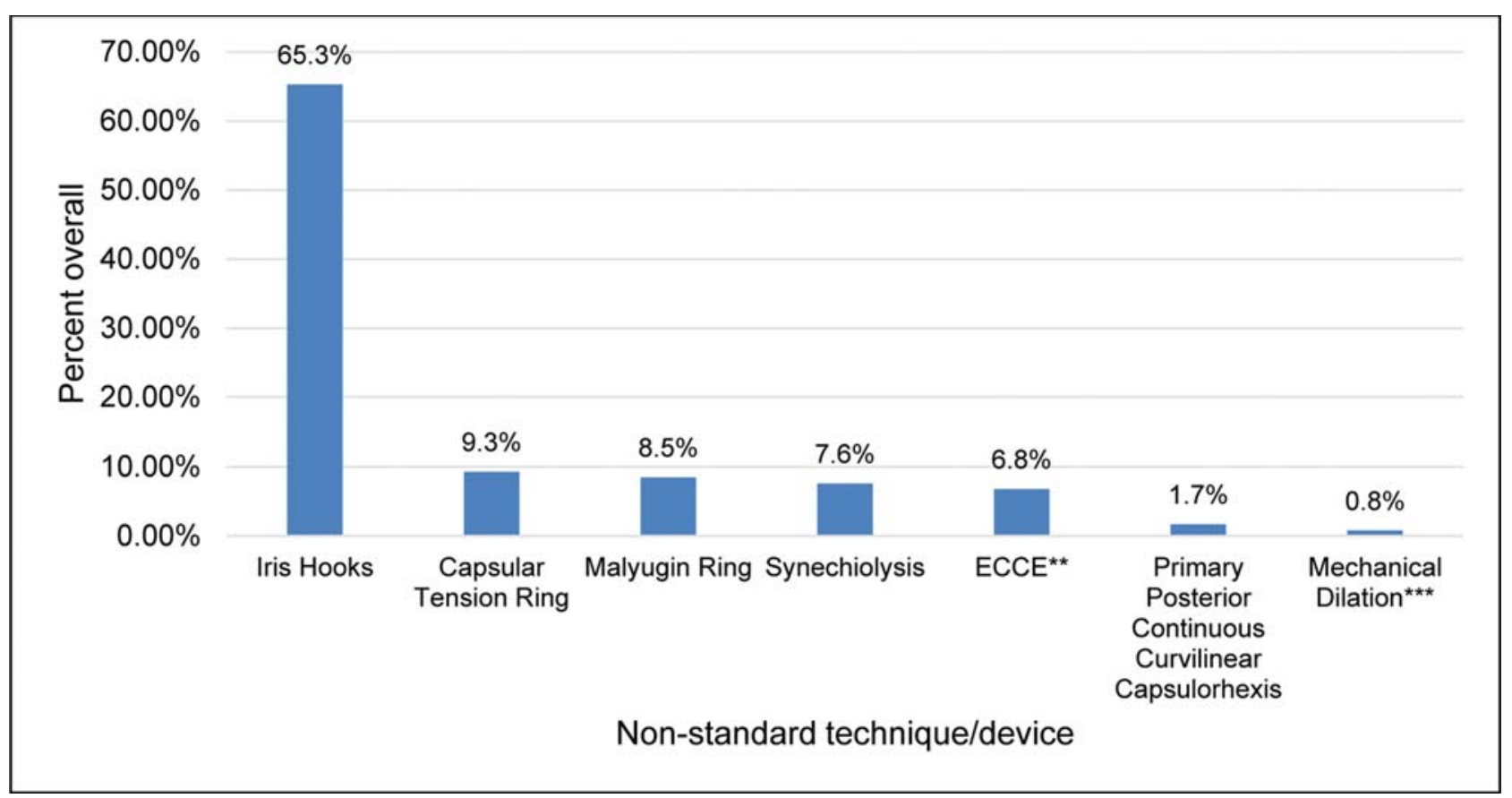

Fig. 1 Bar graph depicting the percentage of utilization of nonstandard technique or device out of the total number of complex cataract surgeries. "Extracapsular cataract extraction. ${ }^{* *}$ Mechanical dilation of iris performed by stretching iris with a second instrument.

number of routine cataract surgeries performed each year was relatively stable from 2008 to 2016 (-Fig. 2). The number of complex cataracts performed over the years was less consistent ranging from 0 in 2007 to 23 in 2015 (-Fig. 3).

\section{Discussion}

ACGME national case logs indicate if a resident's average cataract experience has met the minimum requirements set. However, these data do not indicate the diversity and complexity of the cataract surgery experience. While the ratio of complex to routine cataracts may vary depending on practice population, the average percentage of complex cases coded for Part B Medicare by ophthalmologists in 2017 was $7.6 \%{ }^{8}$ Graduating residents will encounter complex cataracts in practice and exposure to management of these types of cataracts while under the supervision and guidance of an experienced attending is critical to ophthalmology resident training.

A recent survey of young ophthalmologists, either in training or within the first 5 years of practice, revealed that after 10 cases with iris expansion devices or capsular tension rings comfort level plateaued. This suggests that a minimum of 10 cases is required to feel confident using nonstandard techniques or devices. ${ }^{9}$ Our review of third-year resident cataract surgery experience at our institution's VA hospital revealed that complex cataracts constituted a minimal portion of the cases. On average, only $4.5 \%$ of cases were complex. This percentage is surprisingly different from that derived from Medicare claims data. Practicing physicians have monetary incentive to carefully record and document complex cases but residents do not. One reason for the lower incidence of complex cases may be that residents were not properly documenting in the operative report. There are strict requirements for coding complex cataract surgery, and in this study we excluded cases with complications, such as posterior capsule rupture and those requiring trypan blue, other than in the setting of a white or mature cataract, because these surgeries technically do not qualify as complex.

Our review demonstrated that the majority of complex cataract cases experienced by our residents were due to intraoperative floppy iris syndrome or miosis and were addressed using iris hooks. The study revealed that residents had minimal experience with other types of complex cataracts or devices and techniques used to manage these cataracts.

Ideally, graduating residents should be comfortable with a variety of techniques and devices that will allow them to perform complex cases. Ophthalmology is rapidly evolving and continued innovation will lead to more devices for surgical management of cataract. Therefore, a strong foundation in managing diverse cataract cases will equip residents with the skills to incorporate novel techniques and devices into their practice as independent surgeons.

Our findings suggest that attention should be given to ensuring variety in resident case experience. Consideration should be given to adding a complex cataract category to ACGME logs to facilitate tracking of this information and addition of a minimum requirement could also be contemplated. Addition of such a category, with or without minimum requirements, would highlight the importance for both residents and educators alike, of cultivating a diverse surgical case load. When cataract type becomes routinely categorized, it will then be possible to examine the relationship between cataract type (simple or complex) and complication rate, leading to teaching protocols that will optimize patient safety as trainees progress in their training. 


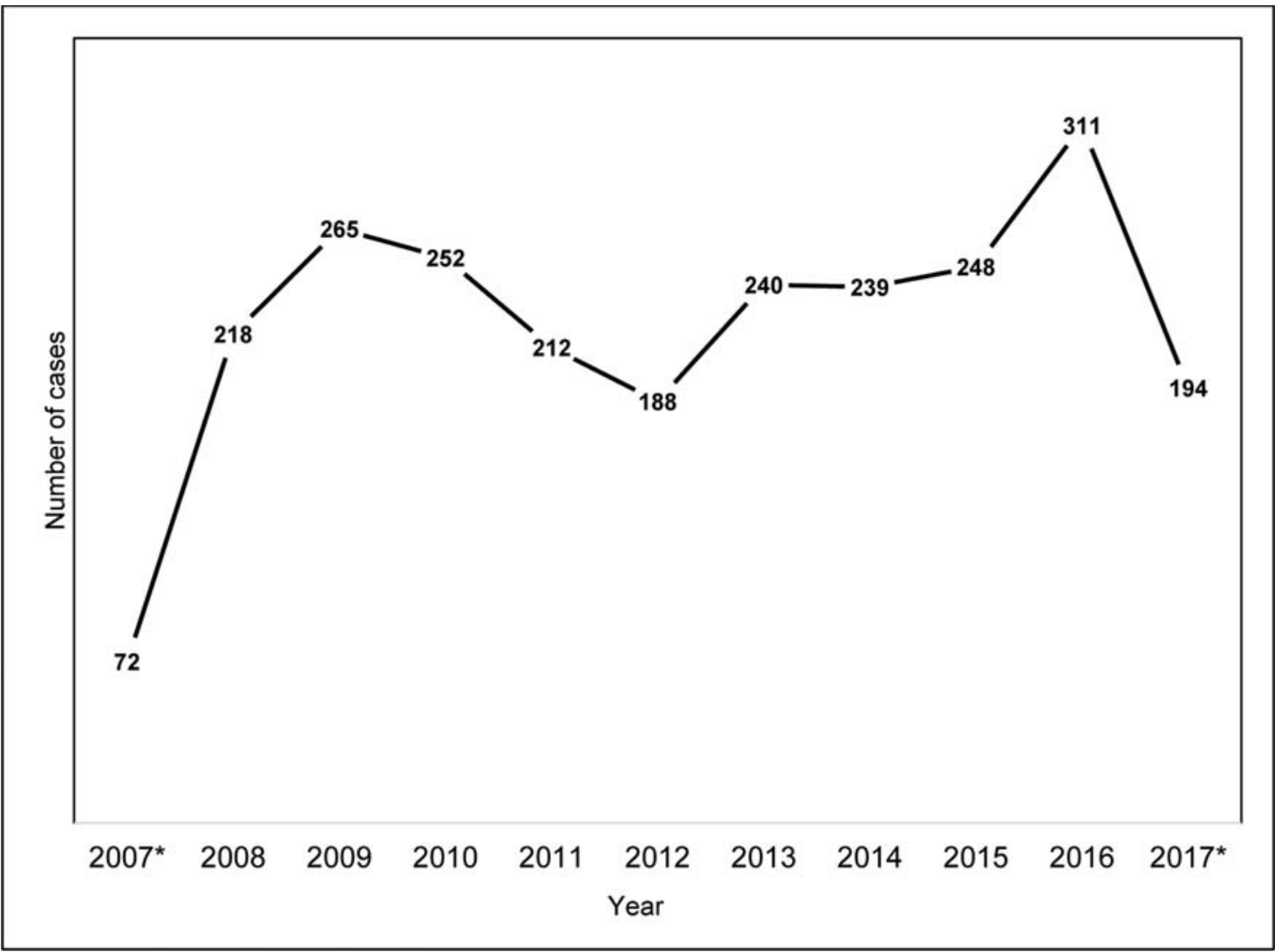

Fig. 2 Line graph depicting the number of routine cataract cases per academic year. "For years 2007 and 2017, only 6 months of data were included, from July to December for 2007 and from January to June for 2017.

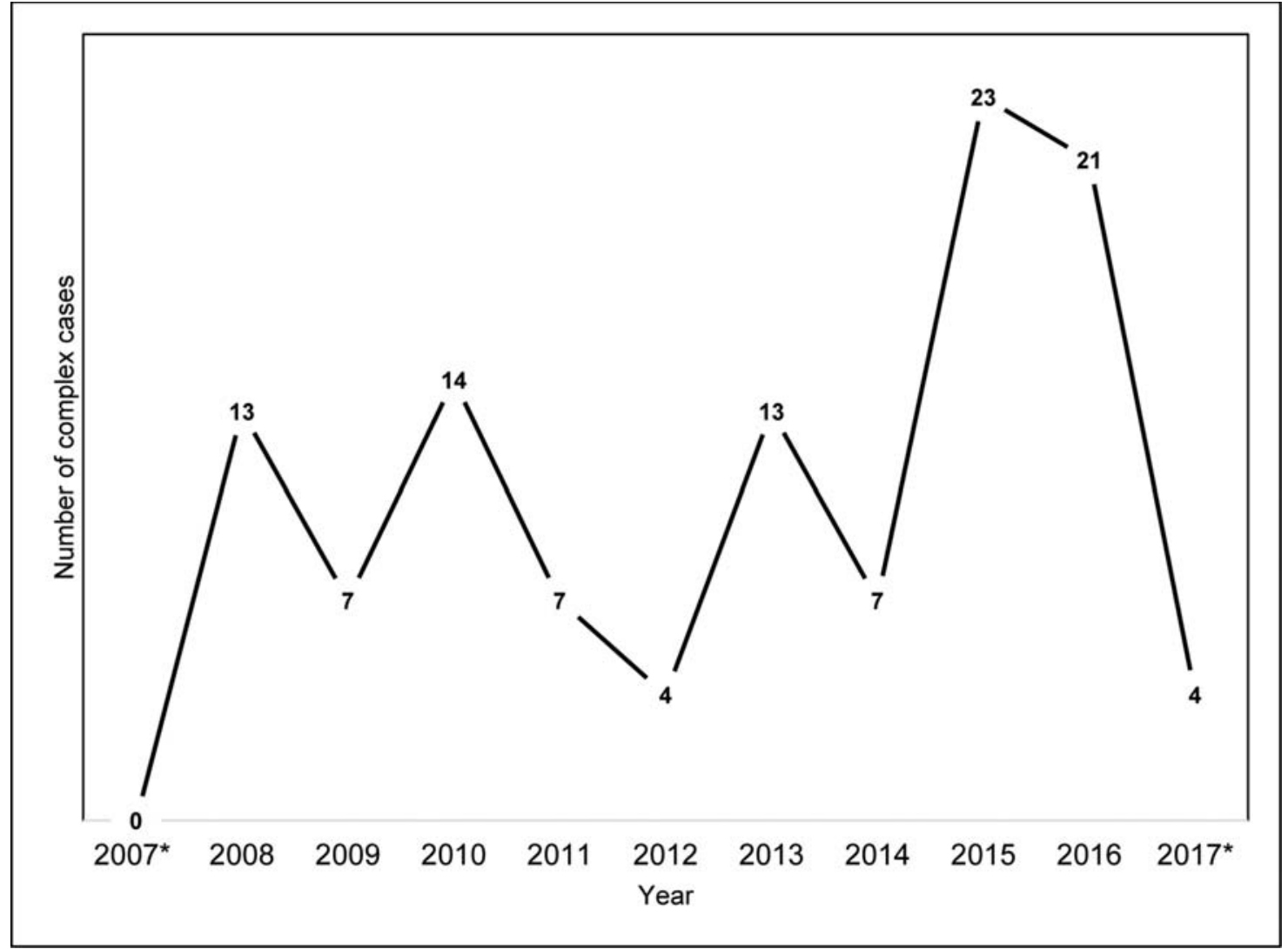

Fig. 3 Line graph depicting the number of complex cataract cases per academic year. For years 2007 and 2017, only 6 months of data were included, from July to December for 2007 and from January to June for 2017. 
The authors recognize this study has limitations. It only evaluated cataract surgery experience at one institution and at a site where approximately $50 \%$ of the surgical experience is obtained. Surgeries during which there was a complication requiring the use of nonstandard techniques or devices were not included in this study as they are technically not complex cataracts. However, it is likely that complications during routine cataract surgeries increase the residents' experience, and thus, we may be underestimating the residents' experience with nonstandard techniques and devices. Nonetheless, our findings underscore a need to evaluate this experience further. From an educational standpoint, it would be informative for other institutions to review their residents' experiences with complex cataract surgeries, to maintain high-quality training experiences.

\section{Conclusion}

Education in cataract surgery should extend beyond achieving minimums, to focus on variety and complexity to optimize training delivered in a competency-based paradigm.

\section{Conflict of Interest}

None declared.

\section{References}

1 Congdon N, O'Colmain B, Klaver CC, et al; Eye Diseases Prevalence Research Group. Causes and prevalence of visual impairment among adults in the United States. Arch Ophthalmol 2004;122 (04):477-485

2 Projections for Cataract. (2010-2030-2050). Available at: https:// nei.nih.gov/eyedata/cataract\#5. Accessed January 13, 2019

3 Cataract surgical rates. Community Eye Health 2017;30(100):88-89

4 Required minimum number of procedures for graduating residents in ophthalmology. Available at: http://www.acgme.org/ Specialties/Documents-and-Resources/pfcatid/13/Ophthalmology. Accessed January 13, 2019

5 Case Logs Statistical Reports. Ophthalmology 2016-2017. Available at: http://www.acgme.org/Data-Collection-Systems/CaseLogs-Statistical-Reports. Accessed July 28, 2019

6 Halm EA, Lee C, Chassin MR. Is volume related to outcome in health care? A systematic review and methodologic critique of the literature. Ann Intern Med 2002;137(06):511-520

7 Bell CM, Hatch WV, Cernat G, Urbach DR. Surgeon volumes and selected patient outcomes in cataract surgery: a populationbased analysis. Ophthalmology 2007;114(03):405-410

8 The 2017 Part B National Summary Data File. Available at: https:// www.cms.gov/Research-Statistics-Data-and-Systems/Downloadable-PublicUse-Files/Part-B-National-Summary-Data-File/Overview.html. Accessed August 4, 2019

9 Schallhorn JM, Ciralsky JB, Yeu E. Resident and young physician experience with complex cataract surgery and new cataract and refractive technology: resultsoftheASCRS 2016 Young EyeSurgeonssurvey. J Cataract Refract Surg 2017;43(05):687-694 